\title{
Smart Driver Assistance System for Accident Forestalling
}

\author{
Shalia ,Kavitha D, Sangeerani Devi A
}

\begin{abstract}
Traffic accidents that happenedaround the worldare increasing a lot. If modern technology is incorporated within vehicle to find the status of the driving person at regular intervals and assist driver about sign boards so that the driver would not lose focal point. The sign board is monitored by using webcam and the text from the image is converted into audio and it directs the driving person. This system observes the heartbeat, sense drowsiness of driver and checks whether the driver has consumed alcohol. If any disaster is noticed, system sends an alert message including the location to the service, sickbay and the person's family members and if there is no serious risk, then the aware message can be ended by the driver in order to avoid wasting the valuable time.
\end{abstract}

IndexTerms-Alertness, sign board, Heart Rate Monitor, optical character recognition, alcohol consumption detection, accident detection.

\section{INTRODUCTION}

Road accidents happened around the world is due to the human error caused by driver which results uncontrollable vehicle condition, therefore life of driver and life of other people are in danger. Traffic police, have to use breath analyzer test for those drivers who are on road for sensing drunk and drive but there is no such system for monitoringstate of driver even though he is driving the automobile. It is also important to know about health condition of the driver through monitoring their heart rate. It is therefore crucial to develop a monitoring method within the vehicle which can keep an eye on the health of the driver while driving and can alert the driver first and, if the driver is not responding, then the situation of driver and accident-prone vehicle on road is informed. Number of accidents can be reduced and thus it can save precious lives and assets of people. Identifying traffic signs have greater effect on driver safety. However, it remains to have some problems. Tracking an object from a moving vehicle is difficult. Visibility of road signs decreases due to air pollution and weather conditions.

Road signs may be affected due to outdoor lighting conditions which varies from day and night. Difficulty is having fast and robust recognition system for identifying road signs. The challenging thing is to find the various road signs trustworthy detection system.

Revised Manuscript Received on October 12, 2019

A.SHALI B.E.,M.E(CSE), Assistant Professor, Departmentof Computer Science and Engineering, Sri SairamEngineering College

D.KAVITHA M, Assistant Professor, Department of Computer Science and Engineering, Sri Sairam Engineering College

A.SANGEERANI DEVIB.Tech(IT),M.E(CSE), Assistant Professor, Department of Computer Scienceand Engineering, Sri Sairam Engineering College
The solution is to discover the automated recognition system that is used to alert a person about the occurrence of indication on the road and to develop a watching system. The proposed way is to incorporate the method based on how the computer obtain information from images.

\section{LITERATURE SURVEY}

[1] Smartphone is one of the most ideal platformsince everyonewill have it and on-board utility are enough to make asystem which detects accident. In a severe case of accident, the Smartphone may be damagedbut the server site is able to analyze the last location before GPS is turned off. Smartphones may also exceed the standard In-Vehicle in certain way such that the smartphone sensors can measure forces closer to those that have been experienced by victims.

[2] Binarization method is used to increaseaccurateness of recognition, it is achieved by applying it before character recognition.Levenshtein distance and F-Measure are used for OCR recognition accuracy so the proposed method reduces computation time. The proposed algorithm outperformsrecognition results as well as processing speedwith Niblack's and Wolf algorithms.

[3] A system that detectseye blinking is designed in such a way that it can monitor the physical state of the person who is driving at usual intervals during driving and, if needed, an alarm is raised to alert the person or vehicle braking system is initiated. Designed system automatically informs the law enforcement authorities about the rogue driver in case of multiple failures. IR sensor is usefulfor night-time environment.

[4] GMR sensor is used in the system and to obtain HR indication, suitable analog digital front-ends is also used. Comprehensive tests were experimented on system prototype to study its feasibility. Accuracy of the developed HR monitor is around $1 \mathrm{bpm}$ which is shown in result. It was also demonstrated that the scheme works well on magneto-plethysmograph. HR indicator works well even when cloth is up to $3.5 \mathrm{~mm}$ thickness. The system is capable of estimating HR variability of an individual.

\section{A. Architecture Diagram}




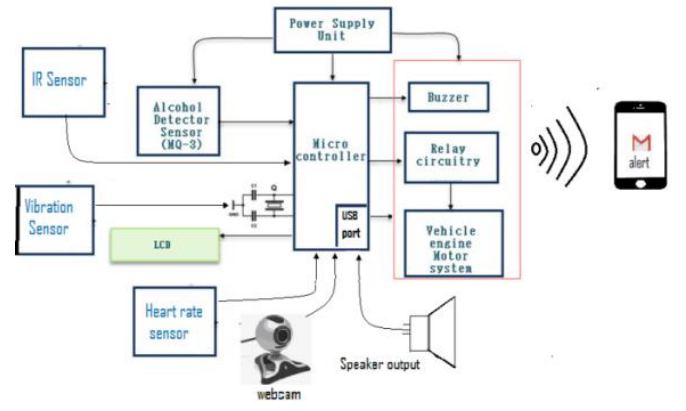

B.Modules Description

- $\quad$ Sign board monitoring

- Accident detection

- Alcohol consumption detection

- $\quad$ Sleep monitoring

- Heart beat monitoring

\section{SIGN BOARD MONITORING:}

The images of the sign boards in the roads are captured through the webcam and converted them into text string using tesseract open source package and then converted into audio output that can be heard by the driver through headset or speaker.

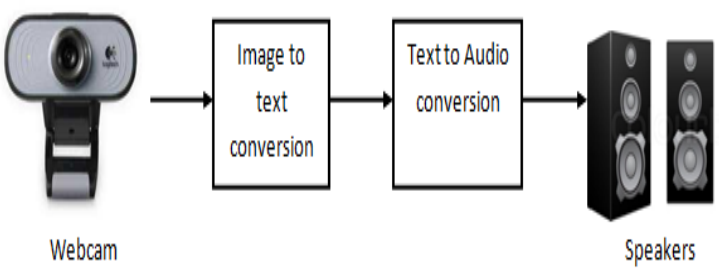

\section{ACCIDENT DETECTION:}

The vibration sensor is connected to the raspberry pi. It detects any vibration that is caused due to the accident and sends the mail alert message through the Ethernet or wifi internet connection to the concerned person's family member or nearby service provider.

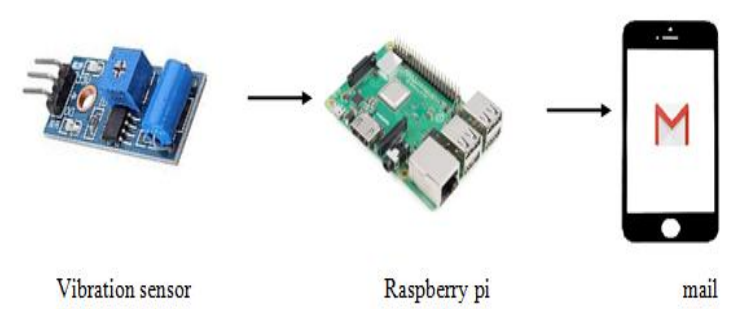

\section{ALCOHOL CONSUMPTION DETECTION}

The alcohol detection sensor is connected to the raspberry pi. It detects the consumption of the alcohol above the threshold range and sends the warning messages through the Ethernet or wifi internet connection to the concerned person's family members or can be customized to apply mechanical brakes.

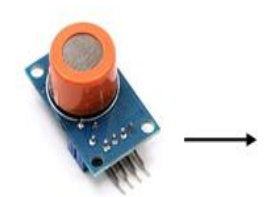

Alcohol detection sensor
Raspberry pi

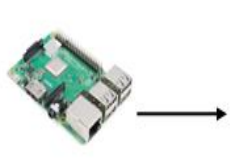

mail

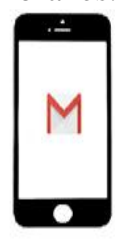

\section{SLEEP MONITORING}

IR sensor is connected to raspberry pi. It exists here to detect the drowsiness of the driving person by monitoring eye blink rate and eye blink pattern. When drowsiness is detected, it sends the warning messages through the Ethernet or wifi internet connection to the concerned person's family members or can be customized to apply mechanical brakes.

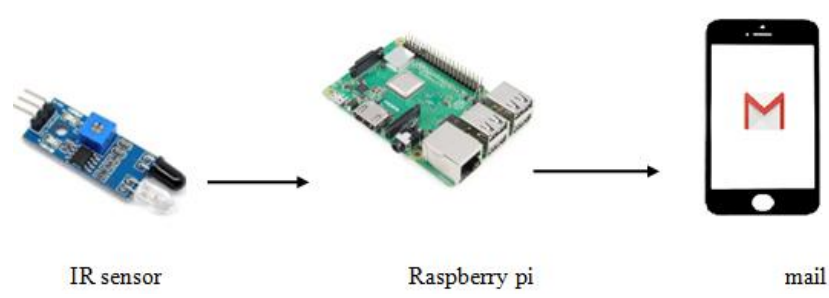

\section{HEART BEAT MONITORING:}

The heartbeat monitoring sensor is connected to raspberry pi. It monitors the heartbeat rate of the driving person and if any abnormal heart beat rate is found, it sends warning messages to the concerned person's family members or can be customized to apply mechanical brakes.

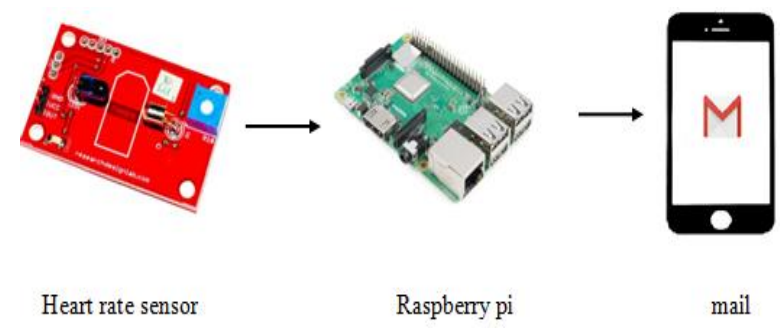

\section{EVALUATION OF SYSTEM}

\subsection{Advantages}

The system provides guidance to the drivers. It also monitors the health condition of the driver. It generates an alarm if the condition of the driver gets worse. This is a real-time monitoring system and user friendly. The vibration sensor, heartbeat monitoring sensor ,IR sensor, alcohol detection sensor, webcam, headset/speakers, power supply , monitor display are integrated into the raspberry pi. The vibration sensor detects any vibration that is caused due to the accident and sends the alert message to the concerned person's family member or nearby sevice provider. The alcohol detection sensor detects the consumption of the alcohol above the threshold range and sends the warning

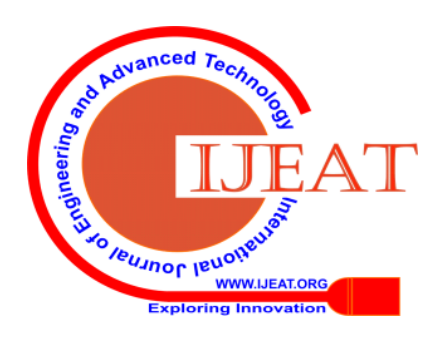


messages to the concerned person's family members or can be customized to apply mechanical brakes. Heartbeat monitoring sensor monitors the heartbeat of the driver and if any deviation is found, it sends the warning messages to the concerned person's family members or can be customized to apply mechanical brakes. The IR sensor is implied here to detect the drowsiness of the driver by monitoring the eye blink rate and eye blink pattern of the driver. The interesting part is the sign board monitoring part which captures the images of the sign boards in the roads and convert them into text string and then convert into audio output that can be heard by the driver through headset or speaker which provides assistance so that the driver would not lose focus on the road.

\subsection{Disadvantages}

The alert mail working largely depends upon the network signal quality. In asevere case of accident, the Smartphone may be damaged but the server site is able to examine the last known location before the GPS is turned off.

\subsection{Applications}

This system can be used extensively in all vehicles. As it is a user-friendly one it can easily assist the drivers. For long distance travels and night travels this plays a vital role as the system itself can guide the driver. It will promotes more security to the passenger and also to the driver as it highly forestalls the accident. The owner can lively monitor the activities of the driving person.

\section{CONCLUSION}

In this paper, a simple and efficient smart driver assistance system was illustrated for the findingof road sign have been anticipated and tested. The attained results show the effectiveness of algorithms and show that this advancement could provide better assistance. On the driver dashboard an eye-blinking detection system can be installed on the driver dashboard, while heartbeat sensor be capable to fit into seat belt and can monitor the driver's physical conditions and provide quick emergency in case of any accidents happened.

\section{FUTURE ENHANCEMENTS}

However, there are some improvements are there for the future. We can improve this prototype further by feeding it more training data so that it could recognize the route boards also. We could customize the system in a way that in case of any accidents to whom should the alert reach first. Also the vehicle collision prevention system which predicts the chances of collision of vehicles in advance can also be integrated with this system to forestall the accidents.

\section{REFERENCES}

1. T. H. Yee and P. Y. Lau, "Mobile vehicle crash detection system," 2018 International Workshop on Advanced Image Technology (IWAIT), Chiang Mai.

2. Lichen Cheng, Lun Li, Jiqiang Liu, ShuoQiu, Wei Wang, Zonghua Zhang,Xiangliang Zhang, "CreditCoin: A Privacy-Preserving Blockchain-Based Incentive Announcement Network for Communications of Smart Vehicles", IEEE Transactions on Intelligent Transportation Systems, 2018.

3. C. H. Mohammed Koya, "An Android Application for Accident Alert", International Journal of Innovative Research in Science Engineering and Technology, vol. 6, no. 5, March 2017.
4. V. LokeshManikanta Kumar, , B. Siva Nagaraju, D. Chandra Sekharand J. Yaswanth Kumar, "Accident Alert using IOT and Android Application", International Journal for Research in Applied Science \& Engineering Technology, vol. 6, no. IV, April 2018.

5. M.H.Awadalla, andT.Jamil,I.Mohammed "Design and implementation of an eye blinking detector system for automobile accident prevention," SoutheastCon 2016, Norfolk, VA.

6. K. Kalyan, V. K. Chugh and C. S. Anoop, "Non-invasive heart rate monitoring system using giant magneto resistance sensor," 2016 38th Annual International Conference of the IEEE Engineering in Medicine and Biology Society (EMBC), Orlando, FL.

7. Amit Meena, SrikrishnaIyer, Monika Nimje, SaketJogJekar, SachinJagtap, Mujeeb Rahman, "Automatic Accident Detection and Reporting Framework for Two Wheelers", IEEE International Conference on Advanced Communication Control and Computing Technologies (ICACCCT), pp. 962-967, May 2014.

\section{AUTHORS PROFILE}

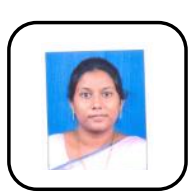

A.SHALI B.E.,M.E(CSE), Assistant Professor, Departmentof Computer Science and Engineering, Sri SairamEngineering College having 10+ years of teachingexperience. My area of interest is Big Data Analytics and Cloud Computing.

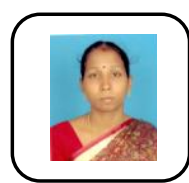

D.KAVITHA M.Tech(CSE), Assistant Professor, Department of Computer Science and Engineering, Sri Sairam Engineering College having 5+ years of teaching experience. My area of interest is Networking and Distributed Systems.

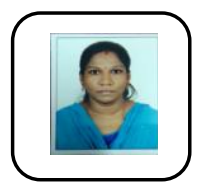

A.SANGEERANI DEVIB.Tech(IT),M.E(CSE), Assistant Professor, Department of Computer Scienceand Engineering, Sri Sairam Engineering Collegehaving 12+ years of teaching experience. My area ofinterest is Data Mining and Big Data Analytics. LifeTime member of ISTE, CSI, published 3 papers Indian Journal of Public Health Research \&amp;Development a scopus indexed journal. 